Original

\title{
Riesgo ergonómico en empresas artesanales del sector de la manufactura, Santander. Colombia
}

\section{Ergonomic risk craft enterprises manufacturing sector, Santander. Colombia}

\section{Claudia Patricia Ardila Jaimes, Reynaldo Mauricio Rodríguez}

Universidad Manuela Beltrán Seccional Bucaramanga. Santander. Colombia

Recibido: 14-12-12

Aceptado: 13-02-13

\section{Correspondencia}

Claudia Patricia Ardila Jaimes

Los Estudiantes, 10-20

Ciudadela Real de Minas

Universidad Manuela Beltrán Seccional Bucaramanga. Colombia.

PBX: (0057) 6525202

Correo electrónico: claudia.ardila@virtualumb.com

Resumen

Introducción: Es importante reconocer que algunas profesiones del ramo de la manufactura no tienen de manera estandarizada programas en ergonomía, por lo tanto se requiere reconocer dichas limitaciones, las cuales podrían desencadenar en patologías musculares, altos índices de ausentismo y disminución en la productividad.

Objetivos: Determinar el perfil de riesgo ergonómico de las empresas artesanales de tabaco y joyería de la región de Santander, Colombia en el año 2010.

Materiales y métodos: Se trató de un estudio descriptivo de corte transversal donde la población encuestada fueron empresas del sector de la manufactura de los sub-sectores artesanales tabacalero y joyero. La unidad de análisis principal fue la empresa. Se diligenció un instrumento que identificaba rasgos de la cultura ergonómica de las empresas.

Resultados: Se encuestaron 15 empresas tabacaleras y 10 joyeras. Las empresas no cuentan con políticas en salud ocupacional en $73 \%$ para el sector tabaco y en $80 \%$ para el sector joyero. En ambos sectores la repetitividad fue el factor ergonómico más prevalente con $80 \%$ y $36,4 \%$ respectivamente. A su vez, $60 \%$ de las empresas del tabaco tienen establecido la elaboración de rediseño de puesto de trabajo, en relación al 10\% presentado por las empresas joyeras, finalmente en ambos sectores se encontró un porcentaje no superior al 13\% sobre realización de capacitaciones sobre control de riesgos ergonómicos a sus trabajadores.

Conclusiones: El perfil de riesgo ergonómico en el sector tabacalero y joyero artesanal se muestra incipiente, considerando estar influenciado por factores como desconocimiento del tema y falta de compromiso gerencial en la implementación de programas que ayuden a minimizar las enfermedades derivadas de las actividades que se desarrollan en cada uno de los sectores.

Med Segur Trab (Internet) 2013; 59 (230) 102-111

Palabras clave: salud ocupacional, ergonomía, joyería artesanal, tabaco artesanal. 


\section{Abstract}

Introduction: It is important to recognize that some professions the manufacturing industry have no standardized way in ergonomics programs therefore requires recognizing these limitations, which could lead to muscle diseases, high rates of absenteeism and decreased productivity.

Objectives: Determine the ergonomic risk profile of the craft and jewelry snuff in the region of Santander, Colombia in 2010.

Methods: This survey was a descriptive cross-sectional study where people were surveyed companies manufacturing sub-sectors of the tobacco and handmade jewelry. The main unit of analysis was the company. He filled out an instrument that identified ergonomic features of the culture of the companies.

Results: We interviewed 15 tobacco companies and 10 jewelers. Companies do not have policies on occupational health in $73 \%$ to snuff sector and $80 \%$ for the jewelery sector. In both sectors the ergonomic factor repeatability was the most prevalent with $80 \%$ and $36.4 \%$ respectively. In turn, $60 \%$ of companies have established snuff redesign making job, compared to $10 \%$ presented by jewelery companies, in both sectors finally found a rate not exceeding $13 \%$ on completion of training on ergonomic risk control their workers.

Conclusions: Ergonomic risk profile ergonomic in the tobacco industry and craft jeweler shows incipient considering be influenced by factors such as ignorance of the subject and lack of management commitment to implement programs to help minimize disease arising from activities taking place in each of the sectors.

Med Segur Trab (Internet) 2013; 59 (230) 102-111

Keywords: occupational health, ergonomics, handmade jewelry, handmade snuff. 


\section{INTRODUCCIÓN}

La Organización Internacional del Trabajo (OIT) enmarca el concepto de ergonomía dentro del espectro de conocimientos y experiencias de las características y capacidades del trabajador, apuntando al uso óptimo del recurso "trabajo humano" haciendo entender que el trabajo llegue a considerarse como más humano. Así mismo, la ergonomía examina no sólo la situación pasiva del ambiente, sino también las ventajas para el operador humano y las aportaciones que pueda hacer, con miras a permitir y fomentar el mejor uso de sus habilidades ${ }^{1}$.

En este mismo contexto, las definiciones de entorno de trabajo saludable han evolucionado durante las últimas décadas, partiendo desde un casi exclusivo enfoque sobre el ambiente físico de trabajo (esquema tradicional de salud y seguridad ocupacional, que consideran los riesgos físicos, químicos, biológicos y ergonómicos) hasta incluir hábitos de estilos de vida saludables, factores psicosociales (organización y cultura de trabajo) y todo aquel rasgo que pueda tener un profundo efecto en la salud del empleado ${ }^{2}$.

El más frecuente e importante campo de investigación donde la ergonomía ha participado ha sido el estudio del desempeño humano frente a las exigencias biomecánicas (postura, fuerza, movimiento) que demandan los puestos de trabajo. A su vez, cuando estos requerimientos sobrepasan la capacidad de respuesta del individuo o no existe una adecuada recuperación biológica de los tejidos, este esfuerzo puede asociarse con el origen o la presencia de trastornos músculo esqueléticos (TME). A su vez, dicho origen es multifactorial, donde la organización del trabajo, la producción, el funcionamiento de la empresa, los procedimientos y los equipos definen el contenido de la actividad en términos de posturas, esfuerzo, repetitividad de movimientos, amplitud articular y duración de los mismos; los cuales generan una carga física que puede desencadenar cuadros reversibles como la fatiga, hasta generar una lesión irreversible ${ }^{3}$.

Por otra parte y para enmarcar el contexto de esta investigación, desde finales de la época colonial y a lo largo del siglo XIX, la producción tabacalera se consideró como de tipo manufacturera, dado que la elaboración del producto era eminentemente manual ${ }^{4}$, con el correr de las décadas este oficio se ha perpetuado a pesar de la inserción de la maquinaria en su proceso. Dentro del mismo contexto manufacturero pero cambiando de subsector, existe el sector productivo de los metales preciosos, en el cual, su última etapa concentra la fabricación de joyas, artículos de orfebrería y bisutería, labor que implica en su mayoría una serie de acciones que involucran el sistema osteo-muscular, siendo predominante el referente a extremidades inferiores y superiores.

Debido a lo anterior, tanto el oficio de la manipulación de tabaco como el oficio de la orfebrería, comparten desde el punto de vista ergonómico ciertos factores de riesgos que pudieran generar trastornos osteo-musculares los cuales conllevan a accidentes laborales o enfermedad profesional que a mediano y largo plazo, se reflejarán en ausentismo laboral y disminución de la productividad.

El Departamento de Santander está ubicado al Oriente de Colombia, tiene una extensión cercana a los $30000 \mathrm{~km}^{2}$ y alberga casi 2`000000 de personas, Bucaramanga es su capital y su área metropolitana la conforma junto a Floridablanca, Piedecuesta y Girón ${ }^{5}$. Dentro de la producción de tabaco artesanal, Santander es responsable de alrededor del $50 \%$ de la producción de tabaco negro del país ${ }^{6}$. Por otra parte, la industria colombiana de metales y piedras preciosas, joyería y bisutería busca consolidarse en los mercados nacional e internacional para el año 2015 y convertirse en el líder latinoamericano en la producción y exportación de artículos de joyería ${ }^{7}$. A su vez, Santander cuenta con cerca de 40 empresas dedicadas a la producción de cigarros como oficio artesanal y existen 150 empresas legalmente constituidas dedicadas a labores de joyería artesanal, las cuales, representan cerca de 300 empleos directos; aunque, se estima que esta cifra es mayor, dado que existen más de 1100 talleres que generan aproximadamente 5.000 empleos directos. 
Desde la mirada de salud ocupacional, tanto los oficios artesanales de fabricación de tabaco como joyería, implican de manera predominante, ejecuciones de movimientos repetitivos de las extremidades superiores y posturas inadecuadas del trabajador, las cuales son situaciones que generan a corto o a largo plazo TME ${ }^{8}$.

Por otra parte, la cultura ergonómica es un tema poco difundido en el contexto nacional y regional, por lo cual es importante resaltar la necesidad de definir su concepto en términos de prevención del riesgo ergonómico y promoción de la salud laboral. Además, siendo estos dos sectores tan prevalentes y productivos en la región y el país, no se dispone de suficiente evidencia científica en cuanto a mediciones del perfil ergonómico, no solo a nivel nacional sino también internacional, principalmente en países afines a la producción de tabaco y joyería artesanal. Por ende, estas mediciones serían un sustento importante para desarrollar planes y estrategias de prevención de accidentes de trabajo, evitar lesiones y enfermedades laborales asociadas al sector manufacturero basados en factores ergonómicos.

\section{MATERIAL Y MÉTODOS}

Se trató de un estudio descriptivo de corte transversal donde la población encuestada fueron empresas del sector de la manufactura de los sub-sectores artesanales tabacalero y joyero. Para este estudio, la unidad de análisis principal fue la empresa como tal, debido a esto, la encuesta fue contestada por el representante legal de la empresa, el representante del departamento de recursos humanos o el encargado del departamento de salud ocupacional. Es importante aclarar, que algunos datos en donde el (n) se refiere a población de trabajadores, son tomados de la información suministrada por el representante de la empresa que contestó el cuestionario basado en información del banco de datos de cada empresa. Inicialmente se indagó por aspectos sociodemográficos de los empleados y posteriormente se diligenció un instrumento que identificaba con 17 preguntas rasgos característicos de la cultura ergonómica de las empresas encuestadas. Así mismo, se efectuó una prueba piloto previa y se tuvieron en cuenta las consideraciones éticas pertinentes.

\section{RESULTADOS}

\section{Aspectos sociodemográficos}

Para efectos de interpretación de los datos, la sigla "tr" se referirá a los trabajadores y la letra "e" a la denominación empresas. De las 15 empresas tabacaleras encuestadas, El $80 \%(n=12 \mathrm{e})$ estaban ubicadas en el municipio de Piedecuesta y de las 10 empresas joyeras, $50 \%$ se localizaron en Bucaramanga. Referente al número de trabajadores por empresa, en el sector tabacalero 8 empresas no superaron los 10 trabajadores, en 5 empresas el número oscilaron entre 11 y 20. Mientras tanto, en el sector de joyería $60 \%$ $(n=6 e)$ no superaron los 10 trabajadores. De acuerdo a la distribución por género, en los dos subsectores estudiados prima el género masculino sobre el femenino, siendo más prevalente en el sector de la joyería artesanal (cuadro 1). En 14 empresas del sector del tabaco se pudo obtener el dato de escolaridad, y de acuerdo a esto, el grupo de mayor frecuencia es el que tiene solo cursada la primaria con $50 \%$, mientras que en el sector joyero, se encontró que $11 \%$ tienen cursada la primaria y como grupo de mayor prevalencia fueron aquellos que habían cursado la secundaria con 77\% (tabla I). 
Tabla I. Variables sociodemográficas en empresas tabacaleras y joyeras

\begin{tabular}{llrrrr}
\hline \multirow{2}{*}{ Variable } & \multirow{2}{*}{ Categorías } & \multicolumn{2}{c}{ Tabaco } & \multicolumn{2}{c}{ Joyas } \\
\cline { 3 - 6 } & & Frecuencia & $\%$ & Frecuencia & $\%$ \\
\hline \multirow{2}{*}{ GÉNERO } & Femenino & 127 & $40,4 \%$ & 35 & $30,0 \%$ \\
& Masculino & 86 & $59,6 \%$ & 105 & $70,0 \%$ \\
\hline \multirow{3}{*}{ ESCOLARIDAD } & Primaria & 82 & $50,6 \%$ & 17 & $11,0 \%$ \\
& Bachillerato & 66 & $40,7 \%$ & 116 & $77,0 \%$ \\
& Pregrado & 10 & $6,2 \%$ & 16 & $11,0 \%$ \\
& Postgrado & 4 & $2,5 \%$ & 1 & $1,0 \%$ \\
\hline
\end{tabular}

\section{Salud ocupacional y ergonomía}

Tanto en el sector tabaco y joyero, la mayoría de trabajadores pertenecen al área operativa, en el sector tabaco $89,2 \%(\mathrm{n}=190 \mathrm{tr})$ son trabajadores operativos mientras que en el sector de la joyería son el $77 \%(\mathrm{n}=115$ tr) (Tabla I). Las empresas del tabaco no cuentan con políticas en salud ocupacional en $73 \%(n=11 e)$, en cuanto al sector joyero, $80 \%(\mathrm{n}=8 \mathrm{e})$ no tienen dichas políticas en su empresa.

De las 15 empresas tabacaleras, todas tienen establecido como control de riesgo ergonómico las pausas de trabajo, a diferencia del sector joyero donde solo $20 \%$ acogen esta norma. De acuerdo al riesgo ergonómico, $80 \%$ de las empresas del tabaco clasifican el riesgo ergonómico de sus trabajadores, mientras que $60 \%$ de las empresas del sector joyero lo hacen. De acuerdo al número de trabajadores, tanto en el sector del tabaco y joyero, la repetitividad es el factor mas prevalente ( $80 \%$ y $36,4 \%$ respectivamente), aunque la diferencia es de un amplio margen (tabla II).

De acuerdo al cuadro 2, la evaluación antropométrica y dimensiones de los puestos de trabajo no es una actividad frecuente que se ejecute, ni siquiera superando el $30 \%$ para cada sector. Por otra parte, de acuerdo a las actividades propias de los trabajadores, las actividades mecánicas son las más ejecutadas siendo cercanas al $94 \%$ en las empresas tabacaleras y del $71 \%$ en el gremio joyero. De acuerdo a la intensidad de la tarea, son livianas o de menor intensidad con $70 \%$ en las empresas del tabaco y con $100 \%$ en el sector joyero.

Tanto en las empresas de tabaco como joyeras, el 100\%: no utilizan métodos estandarizados con el fin de realizar la evaluación ergonómica de las condiciones de trabajo, no cuentan con sistemas de vigilancia epidemiológica para enfermedades osteomusculares y no realizan encuestas de morbilidad sentida de síntomas osteo-musculares. Además, 30\% de las empresas de cada subsector carecen de registro de diagnóstico de las enfermedades profesionales y ausentismo laboral por enfermedades o lesiones osteomusculares.

También se identificó que entre las empresas del tabaco, solo $6,7 \%$ tienen un programa de acondicionamiento físico en relación al $0 \%$ de las empresas joyeras. Así mismo, el $60 \%$ de las empresas del tabaco tienen establecido la elaboración de rediseño de puesto de trabajo como medida de control y mitigación del riesgo ergonómico, en relación al $10 \%$ presentado por las empresas joyeras, finalmente tanto en el gremio tabacalero y joyero se encontró un porcentaje no superior al 13\% en lo que tiene que ver con la realización de capacitaciones sobre control de riesgos ergonómicos a sus trabajadores. 
Tabla II. Variables sobre riesgo ergonómico y aspectos sobre tareas ejecutadas en subsectores tabaco y joyería

\begin{tabular}{llrrrr}
\hline \multirow{2}{*}{ Variable } & \multirow{2}{*}{ Categorías } & \multicolumn{2}{c}{ Tabaco } & \multicolumn{2}{c}{ Joyas } \\
\cline { 3 - 6 } & & Frecuencia & $\%$ & Frecuencia & $\%$ \\
\hline \multirow{2}{*}{ EVALUACIÓN } & Si & 4 & $26,7 \%$ & 1 & $10,0 \%$ \\
ANTROPOMÉTRICA & No & 11 & $73,0 \%$ & 9 & $90,0 \%$ \\
\hline \multirow{2}{*}{ TIPO DE TAREAS } & Mecánicas & 195 & $93,3 \%$ & 107 & $70,9 \%$ \\
EJERCIDAS & Manuales & 7 & $3,3 \%$ & 25 & $16,6 \%$ \\
& Automatizadas & 2 & $1,0 \%$ & 16 & $10,6 \%$ \\
& Mixtas & 5 & $2,4 \%$ & 3 & $2,0 \%$ \\
\hline \multirow{2}{*}{ INTENSIDAD DE LA } & Muy liviano & 2 & $1,0 \%$ & 78 & $53,4 \%$ \\
TAREA & Liviano & 139 & $67,5 \%$ & 68 & $46,6 \%$ \\
& Moderado & 56 & $27,2 \%$ & 0 & $0,0 \%$ \\
& Pesado & 8 & $3,9 \%$ & 0 & $0,0 \%$ \\
EVALUACIÓN DEL & Muy pesado & 1 & $0,5 \%$ & 0 & $0,0 \%$ \\
\hline RIESGO ERGONÓMICO & Si se evalúa & 12 & $80,0 \%$ & 6 & $60,0 \%$ \\
& No se evalúa & 3 & $20,0 \%$ & 4 & $40,0 \%$ \\
\hline \multirow{2}{*}{ RIESGO ERGONÓMICO } & Postura inadecuada & 37 & $17,9 \%$ & 15 & $27,3 \%$ \\
& Vibración & 166 & $80,2 \%$ & 20 & $36,4 \%$ \\
& Temperatura & 4 & $1,9 \%$ & 13 & $23,6 \%$ \\
& & 0 & $0,0 \%$ & 7 & $12,7 \%$ \\
\hline
\end{tabular}

\section{DISCUSIÓN}

Oficios como la producción artesanal de tabaco y joyería tienden a compartir una serie de condiciones físicas, laborales y ergonómicas, por lo tanto es interesante determinar en primera instancia, los aspectos sociodemográficos que pudieran ser diferenciales. En este orden de ideas, se ha considerado que son profesiones que no se les ha otorgado la suficiente relevancia que merece dentro del sector científico ocupacional, debido en parte a catalogarse como un trabajo artesanal realizado por personas de bajos recursos económicos y con un nivel de escolaridad bajo'. Sin embargo, al ser un trabajo manual, el trabajador requiere de agilidad para su elaboración y empaque poniendo en riesgo su salud, exponiéndose constantemente a molestias como dolores musculares, principalmente en cuello, miembro superior y espalda debido a sus movimientos repetitivos y posiciones viciosas $^{10,11}$.

Es importante anotar que desde el punto de vista demográfico, se encontró que la mayoría de empresas en ambos gremios tienen muy poco personal, esto es debido a que en Colombia desde los años mas recientes, el modelo de Fami-empresas (empresas familiares) es una de las principales fuentes de creación de empresas, si bien la subsistencia de las mismas puede resultar compleja, son varios los casos que han traspasado las fronteras y han alcanzado el éxito, por lo tanto, las fami-empresas se vienen consolidando, como una de las salidas a las crisis financieras de las familias a nivel local y nacional ${ }^{12}$.

En cuanto a nivel de escolaridad, se encontró en nuestro estudio que los trabajadores del sector tabaco artesanal tienen una menor escolaridad en relación al sector joyero, sobre todo si se tiene en cuenta el nivel de primaria y bachillerato. Originalmente ambos oficios requieren un entrenamiento importante, $y$ al parecer el oficio de la joyería artesanal tiende a tener movimientos muy elaborados en los cuales se necesita un nivel académico igual o superior al nivel de bachillerato. Lo que si es cierto, es que estas dos profesiones al igual que otras del sector manufacturero como por ejemplo los trabajadores de palma africana y metalmecánica, comparten la característica que pocas personas con estudios 
profesionales están inmersos dentro de la parte operativa y administrativa ${ }^{13,14}$. Por otra parte, un estudio en México sugiere que a medida que en las empresas de la manufactura el nivel académico es elevado, este influye positivamente en la productividad y por consecuencia aporta al incremento de la competitividad dentro del sector ${ }^{15}$.

En materia de políticas en salud ocupacional y teniendo en cuenta la literatura científica, nuestros resultados en cuanto a riesgos ergonómicas no difieren mucho de lo que ocurre en oficios similares como por ejemplo lo visto en talleres de fabricación de diamantes en Israel, donde se revela una carencia de ingeniería de diseño del puesto de trabajo en el tallado, lo que ocasiona dolores de espalda y cuello, además de tensión en el brazo debido a la postura de trabajo. Un análisis de los micro-movimientos y los patrones de movimiento involucrados en esta profesión indican movimientos muy intensos de las manos y los brazos que conllevan una gran aceleración, movimientos rápidos y un alto grado de repetitividad en ciclos muy $\operatorname{cortos}^{16}$.

Por otra parte, en cuanto a prevención de los trastornos osteo-musculares (TOM) se han recomendado ampliamente basarse en medidas preventivas y organizacionales adecuadas, las primeras abarcando el entorno laboral incluyendo el diseño ergonómico de las herramientas, equipos y lugares de trabajo, y las organizacionales centrándose en factores como la formación, instrucción y planes de mejoramiento, todo esto con la finalidad principal de ejecutar un diseño laboral que estribe en adaptar las condiciones de trabajo a la capacidad del trabajador ${ }^{17}$.

En un estudio en una fábrica de tabaco artesanal en Cuba se observó que al mejorar las condiciones de iluminación para realizar el trabajo, se obtuvo una mayor productividad debido al confort del área de trabajo. La iluminación es un factor imprescindible en el micro clima laboral y por otra parte se disminuyó el pago de subsidios por concepto de enfermedades visuales ${ }^{18}$. Dado lo anterior es importante recalcar que no solo el corregir aspectos laborales o ergonómicos mejora o previene patologías musculares sino que también lograría impactar en variables psicológicas y en el clima laboral del empleado y la organización.

En nuestro estudio se notó la carencia de contabilizar el registro de morbilidad osteo-muscular y es importante y necesario tener este tipo de registros, dado que optimiza los recursos en prevención de lesiones de tipo osteo-muscular, lo cual se puede confrontar con un articulo del sector minero artesanal en Paipa Colombia, donde se encontró que la morbilidad auto-reportada ofrece prevalencias altas en trastornos osteo-musculares y antecedentes de lesiones de causa externa por accidente de trabajo, se correspondían con los factores y exposiciones del panorama de riesgos sobre todo del tipo ergonómico (sobre todo en ejecución de tareas repetitivas por periodos prolongados ${ }^{19}$.

En otro estudio en trabajadoras del tabaco artesanal en Brasil, demostró que si bien es cierto ellas son consientes que pueden desarrollar con el tiempo TOM, algunas consideran ocultar su sintomatología por temor a ser despedidas y otras tienden a automedicarse $^{20}$. Teniendo en cuenta este hallazgo es necesario que los programas e intervenciones expliquen y hagan conciencia al trabajador que las normas son para su bienestar y que el propósito no es fiscalizar su labor.

En el sector de la joyería se podría concluir que los riesgos de higiene industrial más acusados se deben a la presencia de numerosos agentes químicos, tales como polvo conteniendo metales, agentes cáusticos y corrosivos; aerosoles; vapores de disolventes. Estos agentes en ocasiones sin contar con algún tipo de medida de control. Por otra parte, es importante informar y capacitar a los trabajadores, en cuanto a los riesgos a los que se someten en sus puestos de trabajo. Lo anterior porque existe un desconocimiento por parte de los empresarios de las obligaciones derivadas de la normatividad vigente en prevención de riesgos laborales, lo cual hace necesaria una mayor divulgación de los riesgos higiénicos presentes en el sector entre los técnicos y profesionales en prevención de riesgos laborales para mejorar las condiciones de trabajo en estas empresas ${ }^{21}$. 
El principio básico de la ergonomía consiste en crear un equilibrio apropiado entre las actividades laborales y la capacidad del trabajador ${ }^{17}$. Las pausas activas hacen parte importante de los principios ergonómicos y se constituyen en un reto en el ámbito de salud laboral, porque el diseño de su aplicación dentro de los programas debe considerar las características particulares de cada proceso de producción. Un estudio consideró que algunos operarios participan en las actividades de pausas activas como un gesto de cooperación y camaradería más que con intención de cuidado ${ }^{22}$. Además, es claro que la evidencia actual apoya la idea que las pausas activas producen cambios en el nivel de actividad física y este a su vez tiene un efecto en la reducción de TOM de miembros superiores $^{23}$. Otra publicación concluye que a pesar de reconocer su importancia para $71 \%$ de trabajadores no realizan pausas activas durante la jornada laboral, además el programa de pausas activas no solo mostró efectos en el largo plazo, sino también en el corto, en la disminución de $\mathrm{TOM}^{24}$. Así mismo, nuestros resultados evidencian que en uno de los dos sectores es mucho más frecuente el uso de esta herramienta, sin embargo es necesario evaluar con más rigurosidad metodológica si en verdad se están efectuando las pausas activas como el protocolo lo dictamina en tiempo lugar y espacio.

Estudios han encontrado como explicación a las limitaciones en la medición de riesgos ergonómicos, la falta de formación y de promoción de una cultura en salud y seguridad en el trabajo, pues prima la idea de que estas situaciones solamente las manejan los que "saben" o "están encargados" del tema en la institución; es decir, que la responsabilidad frente al mismo es única y exclusiva de lo que se conoce como oficina de salud ocupacional y/o de su coordinador ${ }^{25}$. Así mismo se ha demostrado que los programas de vigilancia epidemiológica han demostrado su efectividad en la prevención del desarrollo de enfermedades osteo-musculares entre el personal expuesto a tareas que exigen la realización de movimientos repetitivos de miembros superiores ${ }^{23,26}$. Por lo anterior se sugiere realizar programas de vigilancia epidemiológica centrados en el riesgo más que en la enfermedad involucrando no solo al personal operativo, sino también al administrativo y gerencial.

Desde el punto de vista ergonómico todo oficio, en mayor o menor medida, presenta algún tipo de riesgo. Si bien es cierto en la literatura se encuentran manuales o listas de chequeo para profesiones en particular en términos de riesgo ergonómico, es probable que existan particularidades mas detalladas para el oficio de tabaco y joyería artesanal dentro del rubro empresas familiares ${ }^{27}$. Desde nuestra perspectiva, como alternativas de intervención, se propone involucrar por un lado listas de chequeo exclusivas para estos subsectores y por otro lado se recomienda incluir el concepto de ergonomía participativa la cual se basa en la identificación y prevención del riesgo ergonómico en base a herramientas y protocolos que contemplan la participación directa de empresarios, técnicos y trabajadores para la actuación preventiva ${ }^{28}$.

De acuerdo a la evidencia de efectividad de intervenciones en salud y seguridad ocupacional, se recomienda que emplear únicamente capacitación en levantamiento manual de cargas mostraba resultados inconsistentes, así mismo, la sola capacitación ergonómica se considera como una medida no efectiva al igual que hacer únicamente ajustes ergonómicos en estaciones de trabajo. Dado lo anterior, las intervenciones exitosas son aquellas que buscan combinar varias herramientas y no las aplicadas aisladamente, como por ejemplo los ajustes de la estación de trabajo combinados con capacitación en ergonomía son catalogados como efectivos con evidencia moderada ${ }^{2}$. Por lo anterior se requiere elaborar programas completos y articulados sin dejar de lado ningún detalle en miras a mejorar el panorama de riesgos ergonómicos. Desde nuestros resultados se vislumbra la necesidad de precisar los aspectos legales y reglamentarios de la actividad productiva en el sector artesanal y establecer programas de fomento a largo plazo, situación similar que se observó en un estudio en Colombia en orfebres artesanales ${ }^{29}$.

Con miras hacia el futuro y al revisar las necesidades que se requieren abordar en salud laboral, según el más reciente Congreso mundial sobre seguridad y salud en el trabajo y siguiendo recomendaciones de entes internacionales como la Asociación Internacional Comisión de Salud Ocupacional (ICOH) y la Asociación Internacional de 
Ergonomía (AIE), se considera importante insistir en enfocar la investigación en áreas clave tales como son los aspectos psicosociales, ergonómicos y el impacto de la nanotecnología ${ }^{30}$.

Como conclusiones finales, el perfil de riesgo ergonómico en el sector tabacalero y joyero artesanal se muestra incipiente, considerando que está influenciado por factores como desconocimiento del tema y falta de compromiso gerencial en la implementación de programas que ayuden a minimizar las enfermedades derivadas de las actividades que se desarrollan en cada uno de los sectores ${ }^{10,11}$. De acuerdo a la limitada literatura al respecto de los sectores artesanales estudiados y de acuerdo a los datos encontrados en nuestro estudio, se recomienda realizar estudios que involucren más oficios del sector de la manufactura para realizar y analizar su particularidades en términos de riesgo ergonómico, así mismo se propone diseñar programas específicos en ergonomía para los sectores aquí presentados con miras a implementar y posteriormente evaluar dichas intervenciones con el fin de ser replicadas a nivel regional local e internacional.

\section{AGRADECIMIENTOS}

Este estudio contó con el apoyo de estudiantes de la especialización en Salud Ocupacional de la Universidad Manuela Beltrán Seccional Bucaramanga, quienes aportaron en el desarrollo de la investigación.

Los autores no declaran tener algún conflicto de intereses.

\section{REFERENCIAS BIBLIOGRÁFICAS}

1. OIT Laurig W, Vedder J. Ergonomía, herramientas y enfoques. Volumen 1 Capitulo 29 Enciclopedia de Salud y Seguridad en el trabajo. 1998.

2. OMS. Espacios Laborales Saludables: Fundamentos y Modelo de la OMS. Contextualización, Prácticas y Literatura de Soporte. 2010. ISBN 978924350024 9. (Clasificación NLM: WA 440).

3. Strauss-Gutiérrez A. Guía técnica para el análisis de exposición a factores de riesgo ocupacional en el proceso de evaluación para la calificación de origen de la enfermedad profesional. http://ley100.com/ portal/attachments/153_GUIA_TECNICA_EXPOSICION_FACTORES_RIESGO_OCUPACIONAL.pdf. [En línea] 2011.

4. Teitelbaum V, Gutiérrez F. De la representación a la huelga. Las trabajadoras del tabaco (ciudad de México, segunda mitad del siglo XIX). Boletín Americanista, Año LIX, n. ${ }^{\circ}$ 59, Barcelona, 2009, pp. 265-288, ISSN: 0520-4100.

5. DANE. Proyecciones de Población Colombia. Bogotá: Departamento Administrativo Nacional de Estadística (DANE), 2012.

6. Mojica A, Paredes J. Ensayos sobre economía regional. Características del cultivo del tabaco en Santander. 2005.

7. DPN-Dirección de Planeación Nacional. Documento sectorial metales y piedras preciosas, joyería y bisutería. Agenda interna para la productividad y la competitividad. Bogotá: 2007.

8. OISS-Organización Iberoamericana de Seguridad Social. Trastornos musculoesqueléticos en el ámbito laboral. Incidencia de las condiciones económicas en los accidente de trabajo por sobre esfuerzo en las extremidades superiores. s.l.: 1 edición Mayo, 2011.

9. Delgado L, Durán D, Hernández M, Latorre S. Caracterización del perfil de riesgo ergonómico en las empresas tabacaleras de Santander, Universidad Manuela Beltrán Seccional Bucaramanga. 2011.

10. Correa J, Escobar M, Hernández C. Diagnóstico de la Cultura Ergonómica en las empresas Tabacaleras de Santander. , Universidad Manuela Beltrán. Seccional Bucaramanga. 2011.

11. Corzo N, Mejía O, Nova M, Sierra G. Caracterización del perfil de riesgo ergonómico en las empresas de joyería de Santander. Universidad Manuela Beltrán, Seccional Bucaramanga. 2011.

12. Segovia A, Melgarejo Z. La fami-empresa, un sistema vivo y abierto de desarrollo socioeconómico. s.l.: Tendencias, ISSN-e 0124-8693, vol. 12, n. ${ }^{\circ}$ 2, 2011, págs. 181-199. 
13. Quintero A, Ojeda A, Rangel D. Diagnóstico de la cultura ergonómica en las empresas de siembra de palma africana en Santander. Bucaramanga: Universidad Manuela Beltrán, Seccional Bucaramanga, 2011.

14. Torres B, Pacheco W, Jara F. Caracterización del perfil de riesgo ergonómico de las empresas de metalmecánica de Santander. Trabajo De Investigación Para Optar Al Grado De La Especialidad Salud Ocupacional. Bucaramanga: Universidad Manuela Beltrán, 2011.

15. Rodríguez I, Meza E, Soto E El capital humano y el salario en el nivel de empleo en las empresas manufactureras del Municipio de Tepic. . s.1.: http://xxicolmemeuan.netne.net/extenso/Isidoro_Salvador_ Rodriguez_Vargas_-_el_capital_humano_y_el_salario_en_el_nivel_de_empleo_en_las_empresas_ manufactureras_de_tepic.pdf.

16. Gilad, I, Messe Er.Biomechanics considerations and ergonomic design in diamond polishing. En Advances in Industrial Ergonomics and Safety, dirigido por Kumar. Londres: 1992.

17. OMS. Prevención de trastornos esqueléticos en el lugar de trabajo. Serie protección de la salud de los trabajadores. 2004

18. Águila, A. Propuesta de modificación del sistema de iluminación en el área de clasificado envasado de la fábrica de tabacos para la exportación de Camajuaní. 146, 2011, Observatorio de la Economía Latinoamericana. ISSN 1696-8352.

19. Ospina J, Manrique F, Guío J. Salud y trabajo: minería artesanal del carbón en Paipa, Colombia. av. enferm. [revista en la Internet]. 2010 Jun [citado 2012 Dic 05] ; 28(1): 107-115. Disponible en: http://www. scielo.org.co/scielo.php?script=sci_arttext\&pid=S0121-45002010000100011.

20. Teixeira W, Soares de Freitasc M. NAS MÃOS DAS CHARUTEIRAS, HISTÓRIAS DE VIDA E DE LER/ DORTa. 1, 2011, Revista Baiana de Saúde Pública, Vol. 35.

21. Montero, R. Riesgos Higiénicos en el sector de la Joyería. http://www.insht.es/InshtWeb/Contenidos/ Documentacion/TextosOnline/Rev_INSHT/2001/12/seccionTecTextCompl2.pdf. Centro de Seguridad e Higiene en el Trabajo de Córdoba. 2001.

22. Orozco, Rene Alejandro. Desarrollo de un programa Académico de intervención en el campo de la salud laboral Universidad del Rosario ISSN: 1794-1318 Bogotá 2007 serie documentos borradores de investigación.

23. Buendía E, Ortiz C. Impacto de la implementación de un programa de prevención de lesiones por trauma acumulativo sobre la salud de la población trabajadora del área de empaque y llenado de una empresa farmacéutica de la ciudad de Cali, comparando los años 2006 y 2010. Trabajo de investigación para optar por el postgrado a especialista en administración en salud. Universidad Católica de Manizales 2011.

24. Castro E, Múnera J. E., Sanmartín, M., Valencia, N.A., Valencia, N.D \& González Efectos de un programa de pausas activas sobre la percepción de desórdenes músculo-esqueléticos en trabajadores de la Universidad de Antioquia. Revista educac., E.V. 2011

25. Muñoz A, Castro E. Promoción de la salud en los lugares de trabajo: entre ideal e irreal. Med. segur. trab. [revista en la Internet]. 2010 Dic [citado 2012 Dic 03] ; 56(221): 288-305. Disponible en: http://scielo.isciii. es/scielo.php?script=sci_arttext\&pid=S0465-546X2.

26. Congreso de la República, República de Colombia. Por la cual se modifica el Sistema de Riesgos Laborales y se dictan otras disposiciones en materia de Salud Ocupacional. Ley 1562 2012. Bogotá: s.n., 2012.

27. OIT. Ergonomic checkpoints. Practical and easy-to-implement solutions for improving safety, health and working conditions. OIT; International Ergonomics Association. 2. ${ }^{a}$ ed. Ginebra: OIT, 2010. 303 p. ISBN 9789221226666.

28. Genís-Domenech M. ${ }^{a}$ Susana. Creación de un protocolo para la mejora del puesto de trabajo mediante la Ergonomía Participativa. 2010.

29. Peñas L. Felizzola, O. Orfebres Artesanales de Mompox: Salud Ocupacional y Trabajo. Rev. salud pública [serial on the Internet]. 2001 June [cited 2012 Dec 06] ; 3(2): 143-153. Available from: http://www.scielo. org.co/scielo.php?script=sci_arttext\&pid=S0124-006420010002000.

30. Organization, International Labour. XIX World Congress on Safety and Health at Work: ILO Introductory Report:Global Trends and Challenges on Occupational Safety and Health. Istanbul Turkey, 11-15 September: s.n., 2011. ISBN 978-92-2-125339-6 (print). 